\title{
CSF levels of glutamine synthetase and GFAP to explore astrocytic damage in seronegative NMOSD
}

Iris Kleerekooper, ${ }^{1,2,{ }^{*}}$ Megan K Herbert, ${ }^{3} \mathrm{H}$ Bea Kuiperij, ${ }^{4}$ Douglas Kazutoshi Sato, ${ }^{5,6}$ Kazuo Fujihara, ${ }^{5}$ Dagoberto Callegaro, ${ }^{7}$ Romain Marignier, ${ }^{8}$ Albert Saiz, ${ }^{9}$ Makbule Senel, ${ }^{10}$ Hayrettin Tumani, ${ }^{10,11}$ Brigit A De Jong, ${ }^{12}$ S Anand Trip, ${ }^{1}$ Ichiro Nakashima, ${ }^{5,13}$ Marcel M Verbeek, ${ }^{3,4}$ Axel Petzold ${ }^{14,15}$

\section{Affiliations}

1 Department of Neuroinflammation, University College London, London, UK

2 Department of Neuro-Ophthalmology, Moorfields Eye Hospital, London, UK

3 Department of Neurology and Department of Laboratory Medicine, Radboud University Nijmegen Faculty of Medical Sciences, Nijmegen, The Netherlands

4 Department of Neurology, Radboud University Medical Center, Nijmegen, The Netherlands 5 Department of Neurology, Tohoku University School of Medicine, Sendai, The Japan 6 Brain Institute, Pontificia Universidade Catolica do Rio Grande do Sul, Porto Alegre, Brazil 7 Departamento de Neurologia, Faculdade de Medicina da Universidade de São Paulo, São Paulo, Brazil

8 Service de neurologie, sclérose en plaques, pathologies de la myéline et neuroinflammation, Centre de Référence pour les maladies inflammatoires rares du cerveau et de la moelle (MIRCEM), Hopital Neurologique et Neurochirurgical Pierre Wertheimer, Lyon, France 9 Service of Neurology, Hospital Clinic and Inistitut d'Investigació Biomèdica August Pi i Sunyer (IDIBAPS), Universitat de Barcelona, Barcelona, Spain 10 Department of Neurology, University of Ulm, Ulm, Germany 11 Specialist Clinic of Neurology Dietenbronn, Dietenbronn, Germany 12 Department of Neurology, MS Center Amsterdam, Amsterdam UMC-Locatie VUMC, Amsterdam, The Netherlands

13 Department of Neurology, Tohoku Medical and Pharmaceutical University, Sendai, Japan 14 Institute of Neurology, Neuroimmunology and CSF Laboratory, University College London, London, UK

15 Neurology \& Ophthalmology, VU University Medical Centre, Amsterdam, The Netherlands

*Corresponding author ORCID: 000-0001-6456-9034 E-mail: iris.kleerekooper.18@ucl.ac.uk

To cite: Kleerekooper I, Herbert MK, Kuiperij HB, et al. J Neurol Neurosurg Psychiatry. Epub ahead of print: [please include Day Month Year]. doi:10.1136/jnnp-2019-322286. 


\section{ABSTRACT}

Objective To explore levels of astrocytopathy in neuromyelitis optica spectrum disorder (NMOSD) by measuring levels of the astrocytic enzyme glutamine synthetase (GS) and glial fibrillary acidic protein (GFAP), an established astrocytic biomarker known to be associated with disease activity in multiple sclerosis.

Methods Cerebrospinal fluid concentrations of GS and GFAP were measured by ELISA in patients with NMOSD ( $n=39,28$ aquaporin-4 (AQP4)-Ab-seropositive, 3 double-Abseronegative, 4 myelin oligodendrocyte glycoprotein (MOG)-Ab-seropositive and 4 AQP4-Abseronegative with unknown MOG-Ab-serostatus), multiple sclerosis (MS) ( $n=69$ ), optic neuritis $(n=5)$ and non-neurological controls $(n=37)$.

Results GFAP and GS concentrations differed significantly across groups (both $p<0.001$ ), showing a similar pattern of elevation in patients with AQP4-Ab-seropositive NMOSD. GS and GFAP were significantly correlated, particularly in patients with AQP4-Ab-seropositive NMOSD ( $r s=0.70, p<0.001$ ). Interestingly, GFAP levels in some patients with double-Ab-seronegative NMOSD were markedly increased.

Conclusions Our data indicate astrocytic injury occurs in some patients with double-Abseronegative NMOSD, which hints at the possible existence of yet undiscovered astrocytic autoimmune targets. We hypothesise that elevated GS and GFAP levels could identify those double-Ab-seronegative patients suitable to undergo in-depth autoimmune screening for astrocytic antibodies.

\section{INTRODUCTION}

Neuromyelitis optica spectrum disorder (NMOSD) refers to a heterogenous group of immune mediated CNS diseases that share optic neuritis, transverse myelitis and area postrema syndrome as key clinical features[1].

In recent years understanding of NMOSD has considerably advanced through the identification of two autoimmune targets. The first auto-antibody that was recognised targets aquaporin-4 (AQP4), a water channel which is expressed in astrocytic foot processes[2]. Accordingly, AQP4 targeted autoimmune activity results in profound astrocytopathy, a feature that is distinct from multiple sclerosis $(M S)[3,4]$. The subsequently identified myelinoligodendrocyte glycoprotein (MOG) auto-antibodies are associated with damaged oligodendrocytes and myelin, but do not cause astrocytic injury[5-8].

Identification of these antibodies has facilitated diagnostic procedures, broadened the clinical spectrum and helps guide treatment decisions in $\operatorname{NMOSD}[9,10]$. However, some patients present with a clinical phenotype that is consistent with NMOSD, but do not express AQP4 or MOG antibodies $(A b)[11]$. The response to immunosuppression in these patients suggests a possible autoimmune pathology, but the autoimmune target remains unknown[12]. Here, we aim to investigate astrocytopathy across the full NMOSD spectrum, including double-Abseronegative patients, to gain insight into the pathophysiological processes at play. 
Glial Fibrillary Acidic Protein (GFAP), a part of the astrocyte cytoskeleton, is a very useful biomarker when investigating astrocytic damage[13]. A very substantial increase in cerebrospinal fluid (CSF) GFAP levels during AQP4-Ab-seropositive NMOSD relapses has consistently been reported, and some reports also show low levels of GFAP in MOG-Abseropositive disease[4,6,7,14-18].

One disadvantage of using GFAP as a biomarker, however, is its poor solubility which limits the sensitivity of the test[13]. In order to strengthen the laboratory approach for characterising the widening spectrum of autoimmune astrocytopathies, we have developed a novel assay to detect the predominantly astrocytic enzyme glutamine synthetase (GS)[19]. In contrast to GFAP, GS is highly soluble, facilitating detection[20,21]. We hypothesised that complement mediated damage to astrocytes would not only release GFAP but also GS. Therefore, CSF levels of GS in NMOSD should reveal a similar pattern to what is observed for GFAP[13,22].

In this international multi-centre collaborative study, we used these two astrocytic biomarkers to explore levels of astrocytopathy across the different subgroups of NMOSD. We demonstrate that both GS and GFAP are elevated in AQP4-Ab-seropositive NMOSD and double-Ab-seronegative NMOSD. These data hint at the existence of a yet unidentified astrocytic autoimmune target in a subset of double-Ab-seronegative NMOSD patients.

\section{METHODS}

\section{Patients and non-neurological controls}

This retrospective study included patients with NMOSD, MS and optic neuritis (ON) from five centres (Table S1). Diagnosis of NMOSD was made according to criteria published by Wingerchuk et al. (2015)[1]. We recruited MS patients that had undergone lumbar puncture as part of diagnostic procedure. MS patients were diagnosed according to criteria published by Polman et al. (2005) and all had a long clinical follow-up, during which they displayed a typical disease phenotype[23]. ON patients were diagnosed according to criteria published by Petzold et al.[24] and did not fulfil diagnostic criteria for NMOSD or MS.

Age-matched non-neurological controls were selected from a database of patients referred to the Neurology department in the Radboud University Medical Centre in Nijmegen during the period 2001 to 2009, who underwent lumbar puncture as part of the diagnostic work-up and were confirmed to not have neurological disease. For these patients all routine CSF parameters were normal.

\section{Approvals and consents}

The CSF samples were collected according to standard protocols with local ethics approval[25]. At the time of collection all patients from the Nijmegen centre gave informed consent to lumbar punctures, including later use for scientific purposes but written consent from the patients was legally not required for our analyses. Written informed consent was obtained from all patients from other participating centres.

\section{CSF samples}


CSF samples were collected in polystyrene or polypropylene tubes, centrifuged ( 5 minutes, $860 \mathrm{~g}$ at room temperature), and stored at $-80^{\circ} \mathrm{C}$. For storage purposes, $20 \mathrm{MS}$ samples had been moved to storage at $-20^{\circ} \mathrm{C}$, but not more than six months prior to analysis. Patient information was encoded to maintain confidentiality.

\section{GFAP ELISA}

GFAP levels were measured using a home-made sandwich ELISA (linear up to $250 \mu \mathrm{g} / \mathrm{L}$; interassay variation coefficient $<14 \%$ ) as previously described[26].

For six French patients with NMOSD, GFAP levels have been published previously and sample volumes were too small to repeat the test[27]. Although we excluded these data from analysis, because these data were based on a different method and GFAP levels were not directly comparable, we have displayed these results in Supplementary Figure 1. Because of insufficient CSF material GFAP measurements could not be performed for nine additional subjects (2 AQP4-Ab-seropositive, 2 MOG-Ab-seropositive, 1 double-Ab-seronegative NMOSD, 2 MS patients and 2 controls).

\section{GS ELISA}

GS levels in CSF were measured using our previously published home-made sandwich ELISA incorporating an acidification and neutralisation step for enhanced detection[19].

\section{AQP4-IgG antibody assay}

State of the art cell-based assays were used for AQP4-Ab in the French[28], German[29], Brazilian[30] and Spanish cohorts[31,32].

\section{MOG-IgG antibody assay}

MOG-Ab-serostatus was retrospectively identified from chart study. MOG-IgG Ab status was assessed in local laboratories by cell-based assay.

\section{Statistical analysis}

Categorical variables were described by counts and percentages, and continuous variables by median and interquartile ranges (IQRs). Data were analysed using $\mathrm{R}$ and RStudio. Distribution of age and gender was tested with the Kruskal-Wallis test and Fisher exact test respectively. GS and GFAP levels were compared across groups by the Kruskal-Wallis test. Post-hoc analysis was performed with Dunn Test, with $p$-values adjusted for multiple comparisons with the Benjamini-Hochberg method. Correlations were performed by Spearman's rank analysis $\left(r_{s}=\right.$ Spearman's rho). Multivariate logistic regressions were used to check for distributions of CSF GS and GFAP across groups with two potential confounding factors, age and gender. In the figures GFAP has been log transformed after adding $1(\log 10(G F A P+1))$ for visualisation purposes. Performance of GFAP and GS in discriminating for both NMOSD status and AQP4Ab-seropositive NMOSD status were analysed by plotting receiver operating characteristics (ROC) curves and calculating associated area under curve (AUC) with corresponding 95\% confidence interval $(\mathrm{Cl})$. Optimally effective cut-off values were calculated using the Youden Index.

\section{RESULTS}

\section{Subject characteristics}


We included 39 patients with NMOSD, of which 28 were AQP4-Ab-seropositive, four were MOG-Ab-seropositive, three were double-Ab-seronegative and four were AQP4-Abseronegative but had an unknown MOG-Ab-serostatus. Additionally, 69 patients with MS, five patients with $\mathrm{ON}$ and 37 non-neurological control subjects were included (Table 1). The baseline characteristics are summarised in Table 1. Mean age was comparable between groups ( $p=0.057)$. There was a female predominance in the MS and NMOSD groups compared to controls, as is demographically expected, but this difference did not reach significance $(p=0.051)$. For all NMOSD patients, and for most MS and ON patients, CSF was obtained acutely during a clinical relapse without concomitant treatment. Detailed CSF and clinical data of the double-Ab-seronegative NMOSD subgroup is given in Table S2.

\begin{tabular}{|l|l|l|l|l|l|l|l|l|}
\hline & Controls & MS & $\begin{array}{l}\text { Optic } \\
\text { Neuritis }\end{array}$ & $\begin{array}{l}\text { AQP+ } \\
\text { NMOSD }\end{array}$ & $\begin{array}{l}\text { MOG+ } \\
\text { disease }\end{array}$ & $\begin{array}{l}\text { Double- } \\
\text { Ab neg } \\
\text { NMOSD }\end{array}$ & $\begin{array}{l}\text { Unknown } \\
\text { MOG } \\
\text { NMOSD }\end{array}$ & p-value \\
\hline N & 37 & 69 & 5 & 28 & 4 & 3 & 4 & \\
\hline $\begin{array}{l}\text { Gender } \\
\text { F/M } \\
\text { (\%F) }\end{array}$ & $\begin{array}{l}19 / 18 \\
(51 \%)\end{array}$ & $\begin{array}{l}52 / 17 \\
(75 \%)\end{array}$ & $\begin{array}{l}4 / 1 \\
(80 \%)\end{array}$ & $\begin{array}{l}22 / 5 \\
\left(81 \%^{*}\right)\end{array}$ & $\begin{array}{l}3 / 1 \\
75 \%\end{array}$ & $\begin{array}{l}1 / 2 \\
(33 \%)\end{array}$ & $\begin{array}{l}2 / 2 \\
50 \%\end{array}$ & $>0.05^{\text {a }}$ \\
\hline $\begin{array}{l}\text { Mean } \\
\text { age } \\
\text { (sd) }\end{array}$ & $\begin{array}{l}43.2 \\
(11.1)\end{array}$ & $\begin{array}{l}42.1 \\
(10.6)\end{array}$ & $\begin{array}{l}39.8 \\
(9.5)\end{array}$ & $\begin{array}{l}48.11 \\
(17.5)\end{array}$ & $\begin{array}{l}25.0 \\
(24.1)\end{array}$ & $\begin{array}{l}56.0 \\
(4.4)\end{array}$ & $\begin{array}{l}37.3 \\
(14.0)\end{array}$ & $>0.05^{\mathrm{b}}$ \\
\hline $\begin{array}{l}\text { GFAP } \\
\text { ng/mL } \\
\text { median } \\
\text { (IQR) }\end{array}$ & $\begin{array}{l}0.500 \\
(0.825)\end{array}$ & $\begin{array}{l}0.60 \\
(0.750)\end{array}$ & $\begin{array}{l}0.20 \\
(0.40)\end{array}$ & $\begin{array}{l}5.40 \\
(37.85)\end{array}$ & $\begin{array}{l}0.00 \\
(0.00)\end{array}$ & $\begin{array}{l}76.7 \\
(46.85)\end{array}$ & $\begin{array}{l}0.68 \\
(0.08)\end{array}$ & $<0.001^{\mathrm{b}}$ \\
\hline $\begin{array}{l}\text { GS } \\
\text { Mg/L } \\
\text { median } \\
\text { (IQR) }\end{array}$ & $\begin{array}{l}235.4 \\
(249.0)\end{array}$ & $\begin{array}{l}329.4 \\
(315.0)\end{array}$ & $\begin{array}{l}223.4 \\
(131.0)\end{array}$ & $\begin{array}{l}490.7 \\
(407.2)\end{array}$ & $\begin{array}{l}246.4 \\
(74.0)\end{array}$ & $\begin{array}{l}452.0 \\
(309.0)\end{array}$ & $\begin{array}{l}487.2 \\
(221.2)\end{array}$ & $<0.001^{\mathrm{b}}$ \\
\hline
\end{tabular}

Table 1. Patient demographics and CSF parameters.

${ }^{a}$ Fisher exact test. ${ }^{b}$ Kruskall-Wallis test for non-parametric data. * Gender data missing for 1 AQP4-Ab-seropositive NMOSD patient. GFAP: Glial Fibrillary Acidic Protein; GS: Glutamine Synthetase; MS: multiple sclerosis; NMO+: AQP4-Ab-seropositive NMOSD; NMO-: AQP4-Abseronegative NMOSD; ON: optic neuritis; IQR: interquartile range.

\section{Protein biomarkers}

Neither GS nor GFAP levels were influenced by the storage conditions $\left(-20^{\circ} \mathrm{C}\right.$ versus $-80^{\circ} \mathrm{C}$; $p>0.05)$.

\section{CSF GFAP levels}


Distribution of GFAP levels differed significantly across groups $(p<0.001)$. Median GFAP levels were significantly higher for patients with AQP4-Ab-seropositive NMOSD $(5.40 \mathrm{ng} / \mathrm{mL})$ compared with MS patients $(0.60 \mathrm{ng} / \mathrm{mL}, \mathrm{p}=0.010)$, ON patients $(0.20 \mathrm{ng} / \mathrm{mL}, \mathrm{p}=0.014)$ and non-neurological controls $(0.50 \mathrm{ng} / \mathrm{mL}, \mathrm{p}=0.007)$.

\section{A. Glial Fibrillary Acidic Protein}

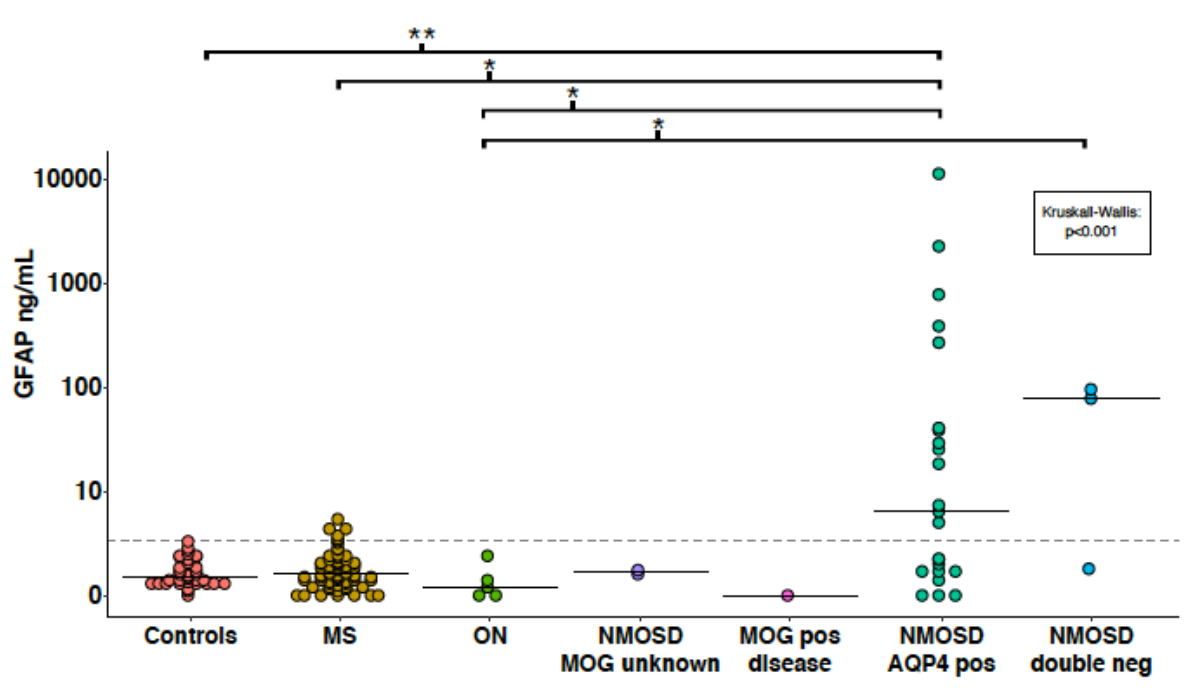

B. Glutamine Synthetase

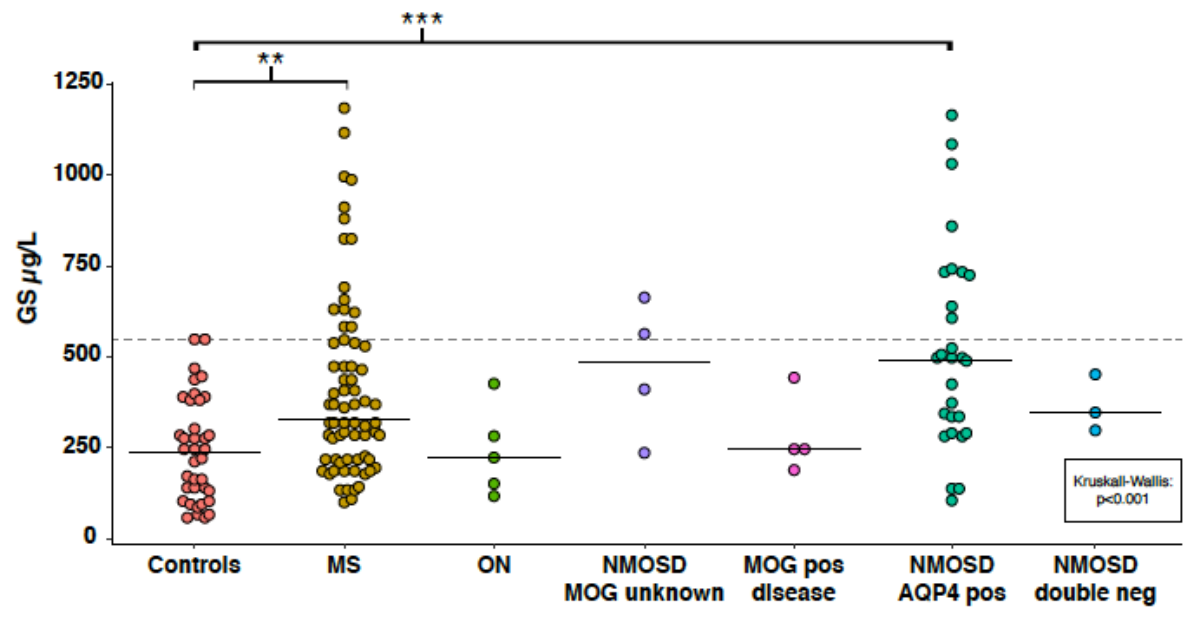

Figure 1. GFAP and GS levels in CSF of controls, MS, ON and NMOSD.

A: CSF GFAP levels in $\mathrm{ng} / \mathrm{mL}$ of non-neurological control subjects, patients with MS, ON and NMOSD (sub-grouped into AQP4Ab-seropositive, MOG-Ab-seropositive, double-Ab-seronegative and AQP4-Ab-seronegative with unknown MOG-Ab serostatus). The Kruskal-Wallis test showed that there was a statistically significant difference of GFAP levels between groups $(p<0.01)$. The grey dotted line represents the highest measured GFAP concentration in non-neurological control subjects. GFAP has been log transformed after adding $1(\log 10($ GFAP+1)) for visualisation purposes.

B: CSF GS levels in $\mu \mathrm{g} / \mathrm{L}$ of non-neurological control subjects, patients with MS, ON and NMOSD (sub-grouped into AQP4-Abseropositive, MOG-Ab-seropositive, double-Ab-seronegative and AQP4-Ab-seronegative with unknown MOG-Ab serostatus). The Kruskal-Wallis test showed that there was a statistically significant difference of GS between groups $(p<0.001)$. The grey dotted line represents the highest measured GS concentration in non-neurological control subjects. 
$*=p<0.05 ; * *=p<0.01 ; * * *=p<0.001 ;$ Controls = non-neurological control subjects; MS = Multiple Sclerosis; NMOSD = neuromyelitis optica spectrum disease; NMO+ = AQP4-Ab-seropositive NMOSD, NMO- = AQP4-Ab-seronegative NMOSD; ON = optic neuritis.

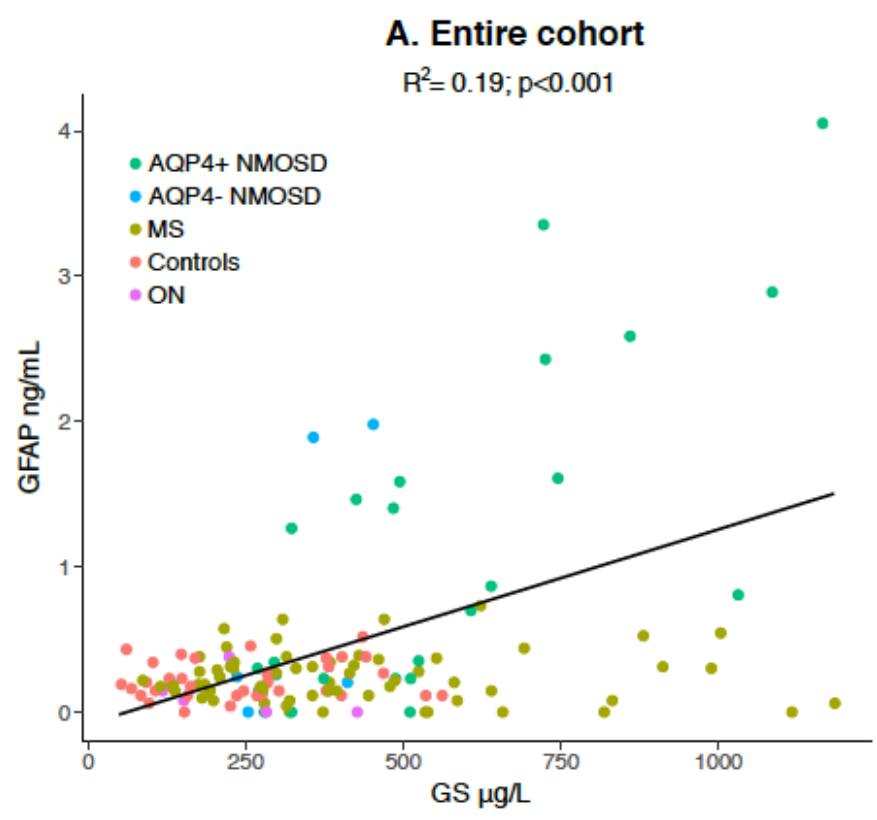

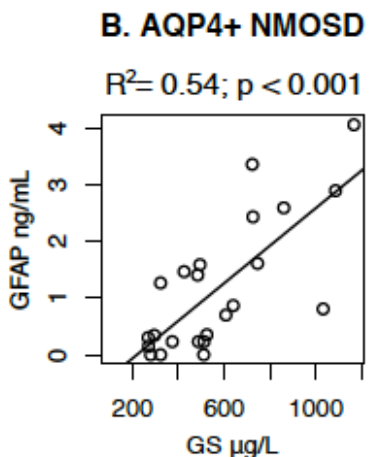

D. MS

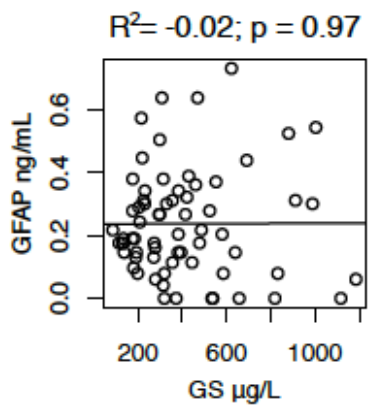

C. AQP4- NMOSD

- double neg

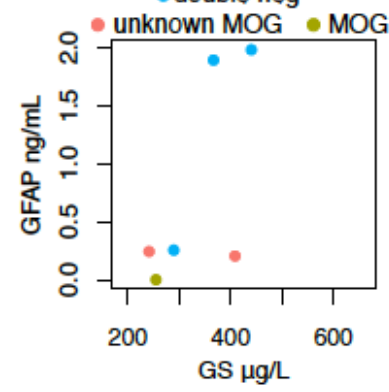

E. Controls

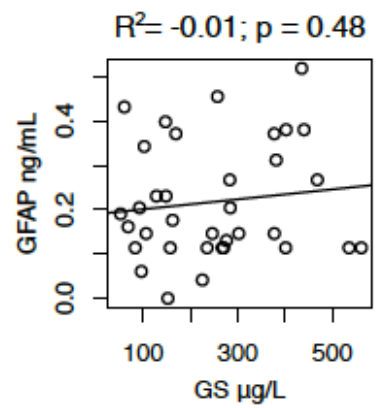

Figure 2. Correlations GFAP and GS.

A. linear regression showing significant positive relationship of GFAP and GS in the entire cohort with colour coding of the dots for the different subgroups (AQP4-Ab-seropositive NMOSD, AQP4-Ab-seronegative NMOSD, multiple sclerosis (MS), optic neuritis $(O N)$ and non-neurological controls. $B$. scatter plot with linear regression line showing the particularly strong positive relationship of GFAP and GS in the AQP4-Ab seropositive NMOSD group. C. colour coded scatter plot of GFAP versus GS levels in the double-Ab-seronegative NMOSD, MOG-Ab-seropositive NMOSD and AQP4-Ab-seronegative with unknown MOG-status cases. This plot is shown for visualisation purposes, even though group sizes are too small to draw conclusions or perform correlation analysis. D. non-significant relationship between GFAP and GS in the multiple sclerosis (MS) cohort. E. non- 
Interestingly, all GFAP concentrations that were substantially higher than the highest measured in non-neurological controls $(2.30 \mathrm{ng} / \mathrm{mL})$ were observed exclusively in AQP4-Abseropositive NMOSD and double-Ab-seronegative NMOSD (Figure 1), although there were six MS patients with a slightly higher GFAP concentration. Furthermore, median GFAP levels for double-Ab-seronegative patients were significantly increased compared with ON patients $(p=0.046)$.

Multivariate logistic regression showed that GFAP levels predicted diagnosis of NMOSD (Beta $=0.461 ; p=0.0257$ ) independent of age and gender (Beta $=-0.011 ; p=0.657$ and Beta=0.238; $p=0.704)$. Additionally, prediction of AQP4-Ab-seropositive NMOSD specifically was nearly significant (Beta $=0.033 ; p=0.060$ ) independent of age and gender (Beta=0.034; $p=0.163$ and Beta=-1.309; $p=0.096$ )

\section{CSF GS levels}

Like GFAP, distribution of GS levels differed significantly across groups ( $p<0.001)$. GS levels were significantly higher for patients with AQP4-Ab-seropositive NMOSD (median $490.7 \mu \mathrm{g} / \mathrm{L}$; $\mathrm{p}<0.001$ ) and MS (median $329.4 \mu \mathrm{g} / \mathrm{L}, \mathrm{p}=0.003$ ) compared with non-neurological controls (median $235.4 \mu \mathrm{g} / \mathrm{L}$ ).

Multivariate logistic regression showed that GS levels predicted diagnosis of NMOSD (Beta=0.002; $p=0.003$ ) independent of age and gender ( $B e t a=0.025 ; p=0.098$ and $B e t a=-0.034$; $p=0.939)$. Furthermore, GS was a significant predictor of AQP4-Ab-seropositive NMOSD (Beta=0.003; $p<0.001$ ) specifically, alongside age (Beta=0.052; $p=0.006)$ but independent of gender (Beta=-0.436; $p=0.442$ ).

Interestingly, we observed an overall positive correlation between GS and GFAP levels $\left(r_{s}=0.23, p<0.001\right)$, which was particularly strong in AQP4-Ab-seropositive NMOSD $\left(r_{s}=0.70\right.$, $p<0.001)$ but absent for the MS group $\left(r_{s}=-0.02, p=097\right)$ and controls $\left(r_{s}=0.07, p=0.48\right)$ (Figure 2.)

\section{Diagnostic performance for astrocytopathy}

The AUC of the ROC curves in discriminating for AQP4-Ab-seropositive NMOSD status was 0.75 (95\% Cl: 0.61-0.89) for GFAP and 0.77 (95\% Cl: 0.67-0.86) for GS. The AUC of the ROC curves for identifying all types of NMOSD were slightly lower for both GFAP $(0.74 ; 95 \% \mathrm{Cl}: 0.61-0.86)$ and GS (0.72; $95 \% \mathrm{Cl}: 0.63-0.82)$ (Figure 3.). The optimally effective cut-off of GFAP concentration to identify AQP4-Ab-seropositive NMOSD was $4.0 \mathrm{ng} / \mathrm{mL}$, with an associated specificity of $97 \%$ and sensitivity of $57 \%$. The optimally effective cut-off for GS was $268.4 \mu \mathrm{g} / \mathrm{L}$, with an associated sensitivity of $89 \%$ and specificity of $41 \%$. The negative predictive value of a GS level lower than $268.4 \mu \mathrm{g} / \mathrm{L}$ was $94 \%$. So, GS can achieve a substantially higher sensitivity but a lower specificity for AQP4-Ab-seropositivity compared with GFAP. This is in line with the observation that all of the 10 AQP4-Ab-seropositive NMOSD patients that had GFAP levels below the identified threshold of $4.0 \mathrm{ng} / \mathrm{mL}$, did have GS levels higher than the threshold of $268.4 \mu \mathrm{g} / \mathrm{L}$. 
A. AQP4-Ab seropositive NMOSD

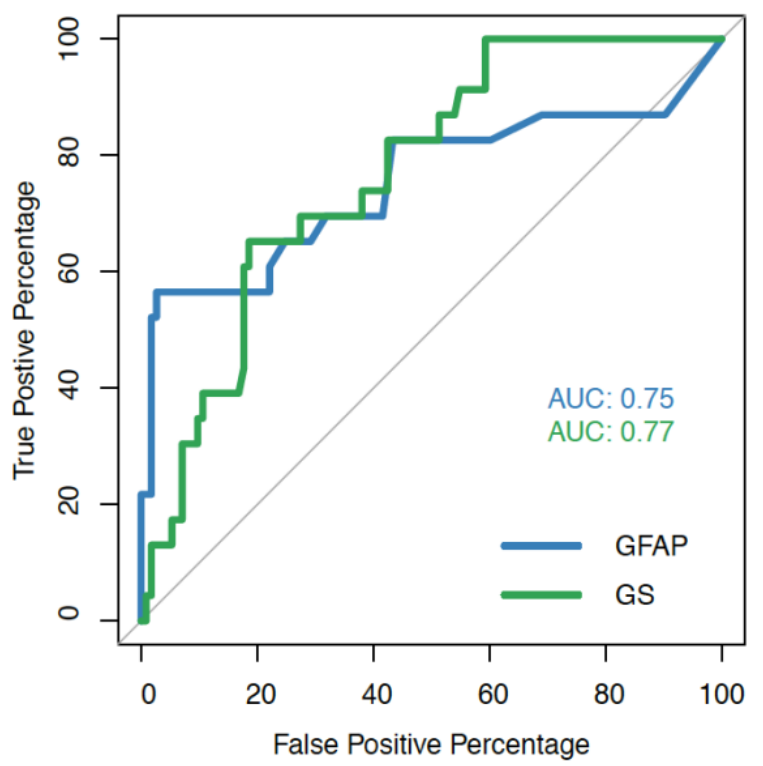

B. NMOSD

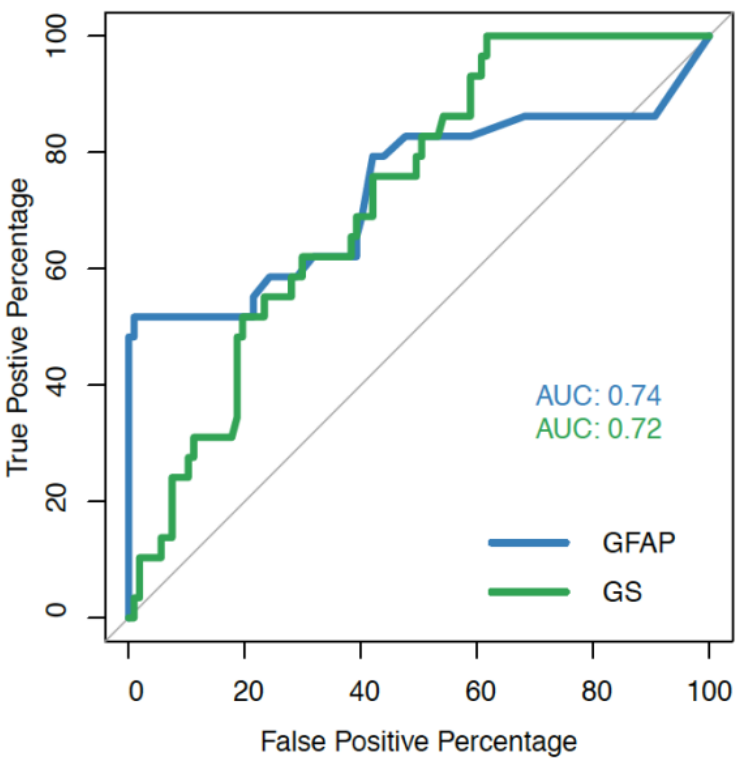

Figure 3. ROC curves GS and GFAP

A. Receiver operating characteristic (ROC) curve of the discriminative performance for the astrocytopathy AQP4-Abseropositive NMOSD of GFAP (blue line) and GS (green line). B. ROC curve of the discriminative performance for NMOSD of GFAP (blue line) and GS (green line).

\section{DISCUSSION}

Here, we report CSF levels of astrocytic biomarkers GS and GFAP in NMOSD, MS, ON and nonneurological controls. Levels of both biomarkers were highest in AQP4-Ab-seropositive NMOSD. The strong correlation between GS and GFAP in AQP4-Ab-seropositive NMOSD suggests that GS is released as a result of astrocytic injury in these patients. GS has a higher sensitivity, but a lower specificity, to astrocytopathy compared with GFAP. Additionally, a subset of double-Ab-seronegative NMOSD cases had substantially increased GFAP levels. This observation suggests astrocytic damage in some double-Ab-seronegative NMOSD patients and hints at the existence of a yet unidentified astrocytic autoimmune target in this group.

Our data replicate prior reports showing that CSF GFAP is increased in AQP4-Ab-seropositive NMOSD but not in MOG-Ab-seropositive NMOSD[4,6,7,14-17,33]. These results are in line with current understanding of NMOSD pathophysiology, as AQP4-Ab-seropositive NMOSD is an autoimmune astrocytopathy, while MOG-Ab-mediated disease results in oligodendrocytic injury but no astrocytopathy[3,9]. Interestingly, we show that very substantial increases of GFAP are not exclusive to AQP4-Ab-seropositive NMOSD, but also occur in double-Abseronegative NMOSD. This observation appears robust as it has been described before[4] and we hypothesise that it hints at the existence of one or more yet unidentified auto-antibodies targeting astrocytes in a subset of double-Ab-seronegative NMOSD patients. A high CSF GFAP concentration may be used to identify those double-Ab-seronegative NMOSD patients with evidence of astrocytopathy that are suitable for in-depth autoimmune screening using labour intensive clonal expansion techniques. Furthermore, double Ab-seronegative NMOSD patients pose a substantial diagnostic challenge, given the lack of a reassuring immunological marker[1]. Diagnosis is based solely on clinical and radiological features, resulting in uncertainty when making treatment decisions. A substantially elevated GFAP in these double- 
Ab negative patients might reinforce diagnosis of $\mathrm{NMO}$ and help guide clinical decision making in some patients.

This is the first study to report on GS levels in NMOSD and related disorders. We showed that CSF levels of this astrocytic enzyme correlate with GFAP levels, especially in AQP4-Abseropositive NMOSD, indicating CSF GS may rise as a result of astrocytic damage, as has been suggested previously[22]. Furthermore, GS and GFAP levels showed a similar pattern of elevation in AQP4-Ab-seropositive NMOSD, while levels in MOG-Ab-seropositive disease appeared to be generally low. However, in contrast to GFAP, GS levels were elevated in MS compared with control subjects as well. This might be because GS, although primarily an astrocytic enzyme, is expressed by oligodendrocytes to some degree as well[34]. Oligodendrocytes are severely damaged in MS and GS immunoreactivity is reduced in MS brain lesions compared with unaffected and control tissues[35]. Additionally, the observed increases of GS in MS and NMOSD patients could partly arise due to leakage of systemic GS across the blood-brain barrier into the CSF, as the blood-brain-barrier is compromised in both disorders. ROC curves of GS and GFAP levels had similar AUCs when testing discriminative performance for AQP4-Ab-seropositive NMOSD. GS provides higher sensitivity compared with GFAP, although specificity for astrocytopathy is relatively low. As GS is elevated more generally in neuroinflammatory disease, it is not a more advantageous diagnostic test for NMOSD compared with GFAP. However, GS appears to be a more sensitive marker of astrocytopathy that could be useful to identify astrocytic injury in seronegative NMOSD patients that fulfil the stringent diagnostic criteria. The high negative predictive value of $94 \%$ associated with GS, suggests that a level below the threshold predicts the absence of astrocytopathy with high accuracy.

The recently described disease entity GFAP autoimmune astrocytopathy may represent the underlying pathophysiologic mechanism in some of the double-Ab-seronegative NMOSD with signs of astrocytopathy[36]. However, currently there is still some uncertainty if GFAP autoimmunity is a primary disease process or a downstream effect of some forms of astrocytic injury[37]. In the future measuring GFAP in the serum may be a less invasive alternative to CSF measurements, as serum GFAP levels have been shown to be increased in NMOSD as well[18]. An important consideration when talking about double-Ab-seronegative NMOSD diagnosis is the possibility of false-negative AQP4- or MOG-Ab results. In this study all antibody assessments were based on highly sensitive cell-based assays, minimising risk of falsenegatives[11].

A limitation of this study was the limited available clinical data, especially regarding details on timing of symptom onset relative to CSF acquisition and disability severity, which we know influences GFAP levels[13]. Furthermore, because CSF volume was limited not all GFAP measurements could be repeated for all patients with the same immunoassay resulting in missing data. Future immunohistochemical analysis of GS and GFAP in NMOSD patients is needed to confirm the potential role of GS as a CSF biomarker for astrocyte injury

In conclusion, our results indicate there may be one or more yet unidentified astrocytic autoimmune targets in double-Ab-seronegative NMOSD patients. We propose that screening of double-Ab-seronegative NMOSD patients for GFAP and GS will identify a subgroup of patients with evidence of astrocytopathy that are suitable for in-depth autoimmune screening 
to identify a possible new astrocytic immune target. GS has suitable properties for screening purposes, while GFAP can be used as a confirmatory test.

\section{ACKNOWLEDGEMENTS}

The authors thank Nathalie Dufay from NeuroBioTec-Banques, (Hospices Civils de Lyon, France) for technical support and the ECTRIMS for supporting Iris Kleerekooper through the Postdoctoral International Research Fellowship.

\section{AUTHOR CONTRIBUTIONS}

$\mathrm{MKH}, \mathrm{IK}$ and AP were responsible for data collection and statistical analysis, interpretation of the data, the drafting and revision of the manuscript, HBK assisted with the revision of the manuscript and reading the manuscript for intellectual content

DKS, KF, DC, RM, AS, HT, BAJ, SAT and IN were responsible for data collection, assisting with interpretation of the data and reading the manuscript for intellectual content. MMV and AP were responsible for the design and conceptualisation of the study and reading the manuscript for intellectual content and revision of the manuscript. All authors commented on the final version of the manuscript.

\section{COMPETING INTERESTS}

IK: received postdoctoral research exchange fellowship from ECTRIMS. MH: no disclosures. BK: no disclosures. DS: has received a scholarship from the Ministry of Education, Culture, Sports, Science and Technology (MEXT) of Japan; a Grants-in-Aid for Scientific Research from the Japan Society for the Promotion of Science (KAKENHI 15K19472); research support from CNPq/Brasil (grant 425331/2016-4), FAPERGS/Ministry of Health/CNPq/SESRS (grant 17/2551-0001391-3) PPSUS/Brazil, TEVA (research grant for EMOCEMP Investigator Initiated Study), and Euroimmun AG (Neuroimmunological Complications associated with Arboviruses); and speaker honoraria from Biogen, Novartis, Genzyme, TEVA, Merck-Serono, Roche, Bayer and has participated in advisory boards from Shire, Roche, TEVA, Merck-Serono and Quest/Athena Diagnostics. AS has received compensation for consulting services and speaker honoraria from Bayer-Schering, Merck-Serono, Biogen-Idec, Sanofi-Aventis, Teva Pharmaceutical Industries Ltd, Novartis and Roche. KF: serves on scientific advisory boards for Bayer Schering Pharma, Biogen Idec, Mitsubishi Tanabe Pharma Corporation, Novartis Pharma, Chugai Pharmaceutical, Ono Pharmaceutical, Nihon Pharmaceutical, Merck Serono, Alexion Pharmaceuticals, Medimmune and Medical Review; has received funding for travel and speaker honoraria from Bayer Schering Pharma, Biogen Idec, Eisai Inc., Mitsubishi Tanabe Pharma Corporation, Novartis Pharma, Astellas Pharma Inc., Takeda Pharmaceutical Company Limited, Asahi Kasei Medical Co., Daiichi Sankyo, and Nihon Pharmaceutical; serve as an editorial board member of Clinical and Experimental Neuroimmunology (2009-present) and an advisory board member of Sri Lanka journal of Neurology; has received research support from Bayer Schering Pharma, Biogen Idec Japan, Asahi Kasei Medical, The Chemo-SeroTherapeutic Research Institute, Teva Pharmaceutical, Mitsubishi Tanabe Pharma, Teijin Pharma, Chugai Pharmaceutical, Ono Pharmaceutical, Nihon Pharmaceutical, and Genzyme Japan; is funded as the secondary investigator (\#22229008, 2010-2015) by the Grants-in-Aid for Scientific Research from the Ministry of Education, Science and Technology of Japan and as the secondary investigator by the Grants-in-Aid for Scientific Research from the Ministry of Health, Welfare and Labor of Japan (2010-present). DC: no disclosures. RM: no disclosures. 
AS: has received compensation for consulting services and speaker honoraria from BayerSchering, Merck-Serono, Biogen-Idec, Sanofi-Aventis, Teva Pharmaceutical Industries Ltd, Novartis and Roche. MS: has received consulting and speaker honoraria as well as travel reimbursements from Bayer, Biogen, Celgene, Roche, Sanofi Genzyme and TEVA and research funding from the Hertha-Nathorff-Program and University of UIm. HT: received funding for research projects, lectures and travel from Bayer, Biogen, Genzyme, Fresenius, Merck, Mylan, Novartis, Roche, Siemens Health Diagnostics, Teva, and received research support from Hertie-Stiftung, BMBF, University of Ulm and Landesstiftung BW. BJ: B.A. de Jong has received speaker and consulting fees from Merck-Serono, Biogen, TEVA, Genzyme and Novartis. SAT: non-financial support and/or personal fees from Biogen, Merck Serono, Novartis, Roche and Teva. Involved with clinical trials run by Biogen and Sanofi Genzyme. Supported by the National Institute for Health Research (NIHR) University College London Hospitals (UCLH) Biomedical Research Centre. IN: Dr. Nakashima reports personal fees from Mitsubishi Tanabe Pharma, personal fees from Biogen Japan, personal fees from Takeda Pharmaceuticals, personal fees from Novartis Pharmaceuticals, grants from LSI Medience, grants from Ministry of Education, Science and Technology of Japan, and the Ministry of Health, Welfare and Labor of Japan. MV: received grants from ZonMw, Alzheimer Nederland, Weston Brain Institute, NIH. AP: member of the steering committee for the OCTiMS study (Novartis), no consulting fees. Performs OCT QC for the Passos study (Novartis), received consulting fees. Received speaker fees from Heidelberg Engineering.

\section{FUNDING}

Study funding: This study was supported in part by Marató de TV3 (20141830, AS).

\section{REFERENCES}

1 Wingerchuk D, Banwell B, Bennett JL, et al. International consensus diagnostic criteria for neuromyelitis optica spectrum disorders. Neurology 2015;85:177-89. doi:10.1212/WNL.0000000000001729

2 Lennon VA, Wingerchuk DM, Kryzer TJ, et al. A serum autoantibody marker of neuromyelitis optica: distinction from multiple sclerosis. Lancet 2004;364:2106-12.

3 Papadopoulos M, Verkman A. Aquaporin 4 and neuromyelitis optica. Lancet Neurol 2012;11:535-44. doi:10.1016/S1474-4422(12)70133-3

4 Wei Y, Chang H, Li X, et al. CSF-S100B Is a Potential Candidate Biomarker for Neuromyelitis Optica Spectrum Disorders. Biomed Res Int 2018;2018:5381239. doi:10.1155/2018/5381239

5 Sato DK, Callegaro D, Aurelio Lana-Peixoto M, et al. Distinction between MOG antibody- positive and AQP4 antibody-positive NMO spectrum disorders. Neurology 2014;82:474-81. doi:10.1212/WNL.0000000000000101

$6 \quad$ Ikeda K, Kiyota N, Kuroda H, et al. Severe demyelination but no astrocytopathy in clinically definite neuromyelitis optica with anti-myelin-oligodendrocyte glycoprotein antibody. Mult Scler J 2015;21:656-9. doi:10.1177/1352458514551455

$7 \quad$ Kaneko K, Sato D, Nakashima I, et al. Myelin injury without astrocytopathy in neuroinflammatory disorders with MOG antibodies. J Neurol Neurosurg Psychiatry 2016;87:1257-9.

8 Wang JJ, Jaunmuktane Z, Mummery C, et al. Inflammatory demyelination without astrocyte loss in MOG antibody-positive NMOSD. Neurology 2016;87:229-31.

doi:10.1212/WNL.0000000000002844 
9 Fujihara K. Neuromyelitis optica spectrum disorders: still evolving and broadening. Curr Opin Neurol 2019;32:385-394. doi:10.1097/WCO.0000000000000694

10 Pittock S, Verthele K, Fujihara K, et al. Eculizumab in Aquaporin-4-Positive Neuromyelitis Optica Spectrum Disorder. N Engl J Med 2019; [Epub ahea. doi:10.1056/NEJMoa1900866

11 Jiao Y, Fryer JP, Lennon VA, et al. Updated estimate of AQP4-IgG serostatus and disability outcome in neuromyelitis optica. Neurology 2013;81:1197-204.

12 Mealy MA, Kim S, Schmidt F, et al. Aquaporin-4 serostatus does not predict response to immunotherapy in neuromyelitis optica spectrum disorders. Mult Scler J 2018;24:173742. doi:10.1177/1352458517730131

13 Petzold A. Glial fibrillary acidic protein is a body fluid biomarker for glial pathology in human disease. Brain Res 2015;1600:17-31. doi:10.1016/j.brainres.2014.12.027

14 Misu T, Takano R, Fujihara K, et al. Marked increase in cerebrospinal fluid glial fibrillar acidic protein in neuromyelitis optica: an astrocytic damage marker. J Neurol Neurosurg Psychiatry 2009;80:575-7. doi:10.1136/jnnp.2008.150698

15 Petzold A, Pittock S, Lennon V, et al. Neuromyelitis optica-lgG (aquaporin-4) autoantibodies in immune mediated optic neuritis. J Neurol 2010;81:109-11. doi:10.1136/jnnp.2008.146894

16 Takano R, Misu T, Takahashi T, et al. Astrocytic damage is far more severe than demyelination in NMO: a clinical CSF biomarker study. Neurology 2010;75:208-16. doi:10.1212/WNL.0b013e3181e2414b

17 Wei Y, Chang H, Li X, et al. Cytokines and Tissue Damage Biomarkers in First-Onset Neuromyelitis Optica Spectrum Disorders: Significance of Interleukin-6.

Neuroimmunomodulation 2018;25:215-24. doi:10.1159/000494976

18 Watanabe M, Nakamura Y, Michalak Z, et al. Serum GFAP and neuro fi lament light as biomarkers of disease activity and disability in NMOSD. Neurology 2019; [Epub ahea. doi:10.1212/WNL.0000000000008160

19 Herbert MK, Kuiperij HB, Verbeek MM. Optimisation of the quantification of glutamine synthetase and myelin basic protein in cerebrospinal fluid by a combined acidification and neutralisation protocol. J Immunol Methods 2012;381:1-8.

doi:10.1016/j.jim.2012.04.001

20 Tumani $H$, Shen GQ, Peter JB. Purification and immunocharacterization of human brain glutamine synthetase and its detection in cerebrospinal fluid and serum by a sandwich enzyme immunoassay. J Immunol Methods 1995;188:155-63.

21 Bernstein H, Bannier J, Meyer-Lotz G, et al. Distribution of immunoreactive glutamine synthetase in the adult human and mouse brain. Qualitative and quantitative observations with special emphasis on extra-astroglial protein localization. J Chem Neuroanat 2014;6162:33-50. doi:10.1016/j.jchemneu.2014.07.003

22 Marignier R, Nicolle A, Watrin C, et al. Oligodendrocytes are damaged by neuromyelitis optica immunoglobulin G via astrocyte injury. Brain 2010;133:2578-91. doi:10.1093/brain/awq177

23 Polman CH, Reingold SC, Edan G, et al. Diagnostic Criteria for Multiple Sclerosis: 2005 Revisions to the "McDonald Criteria". Ann Neurol 2005;58:840-6. doi:10.1002/ana.206703 24 Petzold A, Wattjes MP, Costello F, et al. The investigation of acute optic neuritis: A review and proposed protocol. Nat Rev Neurol 2014;10:447-58.

doi:10.1038/nrneurol.2014.108 
25 Teunissen CE, Petzold A, Bennett J, et al. A consensus protocol for the standardization of cerebrospinal fluid collection and biobanking. Neurology 2009;73:191422. doi:10.1212/WNL.0b013e3181c47cc2

26 Geel WJA Van, Reus HPM De, Nijzing $\mathrm{H}$, et al. Measurement of glial fibrillary acidic protein in blood: an analytical method. Clin Chim Acta 2002;326:151-4.

27 Petzold A, Marignier R, Verbeek $M$, et al. Glial but not axonal protein biomarkers as a new supportive diagnostic criteria for Devic neuromyelitis optica? Preliminary results on 188 patients with different neurological diseases. J Neurol Neurosurg Psychiatry 2011;82:467-9. doi:10.1136/jnnp.2009.196550

28 Marignier R, Berard-Valnet R, Giraudon $\mathrm{P}$, et al. Aquaporin-4 antibody-negative neuromyelitis optica. Distinct assay sensitivity-dependent entity. Neurology 2013;80:2194200. doi:10.1212/WNL.0b013e318296e917

29 Waters $\mathrm{P}$, Reindl M, Saiz A, et al. Multicentre comparison of a diagnostic assay: Aquaporin-4 antibodies in neuromyelitis optica. J Neurol Neurosurg Psychiatry 2016;87:1005-15. doi:10.1136/jnnp-2015-312601

30 Sato DK, Callegaro D, Jorge FMDH, et al. Cerebrospinal Fluid Aquaporin-4 Antibody Levels in Neuromyelitis Optica Attacks. Ann Neurol 2014;76:305-9. doi:10.1002/ana.24208 31 Höftberger R, Sepulveda M, Armangue T, et al. Antibodies to MOG and AQP4 in adults with neuromyelitis optica and suspected limited forms of the disease. Mult Scler 2015;21:866-74. doi:10.1177/1352458514555785

32 Höftberger R, Sabater L, Marignier R, et al. An Optimized Immunohistochemistry Technique Improves NMO-IgG Detection: Study Comparison with Cell-Based Assays. PLoS One 2013;8:6-11. doi:10.1371/journal.pone.0079083

33 Petzold A, Woodhall M, Khaleeli Z, et al. Aquaporin-4 and myelin oligodendrocyte glycoprotein antibodies in immune-mediated optic neuritis at. J Neurol Neurosurg Psychiatry 2019;0:1-6. doi:10.1136/jnnp-2019-320493

34 D'Amelio F, Eng LF, Gibbs MA. Glutamine Sgnthetase Immunoreactivity is Present in Oligodendroglia of Various Regions of the Central Nervous System. Glia 1990;3:335-41. 35 Werner P, Pitt D, Raine CS. Multiple Sclerosis: Altered Glutamate Homeostasis in Lesions Correlates with Oligodendrocyte and Axonal Damage. Ann Neurol 2001;50:169-80.

36 Fang B, McKeon A, Hinson S, et al. Autoimmune Glial Fibrillary Acidic Protein Astrocytopathy: A Novel Meningoencephalomyelitis. JAMA Neurol 2016;73:1297-307. doi:10.1001/jamaneurol.2016.2549

37 Zekeridou A, McKeon A, Flanagan E. A path to understanding autoimmune GFAP astrocytopathy. Eur J Neurol 2018;25:421-2. doi:10.1111/ene.13527 